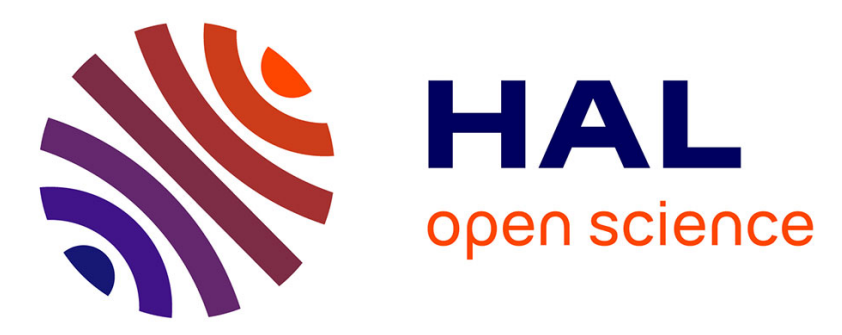

\title{
Binary mixtures of disks and elongated particles: Texture and mechanical properties
}

Emilien Azéma, Itthichai Preechawuttipong, Farhang Radjai

\section{To cite this version:}

Emilien Azéma, Itthichai Preechawuttipong, Farhang Radjai. Binary mixtures of disks and elongated particles: Texture and mechanical properties. Physical Review E , 2016, 94 (4), pp.42901. 10.1103/PhysRevE.94.042901 . hal-01382536

\section{HAL Id: hal-01382536 \\ https://hal.science/hal-01382536}

Submitted on 17 Oct 2016

HAL is a multi-disciplinary open access archive for the deposit and dissemination of scientific research documents, whether they are published or not. The documents may come from teaching and research institutions in France or abroad, or from public or private research centers.
L'archive ouverte pluridisciplinaire HAL, est destinée au dépôt et à la diffusion de documents scientifiques de niveau recherche, publiés ou non, émanant des établissements d'enseignement et de recherche français ou étrangers, des laboratoires publics ou privés. 


\title{
Binary mixtures of disks and elongated particles: Texture and mechanical properties
}

\author{
Emilien Azéma, ${ }^{1,2, *}$ Itthichai Preechawuttipong, ${ }^{2, \dagger}$ and Farhang Radjai ${ }^{1,3, \ddagger}$ \\ ${ }^{1}$ Laboratoire de Mécanique et Génie Civil (LMGC), Université de Montpellier, CNRS, Montpellier, France \\ ${ }^{2}$ Department of Mechanical Engineering, Faculty of Engineering, Chiang Mai University, 239 Huay Kaew Rd., Chiang Mai 50200, Thailand \\ ${ }^{3}\langle M S E\rangle^{2}$, UMI 3466 CNRS-MIT, MIT Energy Initiative, 77 Massachusetts Avenue, Cambridge, Massachusetts 02139, USA
}

(Received 23 June 2016; published 14 October 2016)

\begin{abstract}
We analyze the shear strength and microstructure of binary granular mixtures consisting of disks and elongated particles by varying systematically both the mixture ratio and degree of homogeneity (from homogeneous to fully segregated). The contact dynamics method is used for numerical simulations with rigid particles interacting by frictional contacts. A counterintuitive finding of this work is that the shear strength, packing fraction, and, at the microscopic scale, the fabric, force, and friction anisotropies of the contact network are all nearly independent of the degree of homogeneity. In other words, homogeneous mixtures have the same strength properties as segregated packings of the two particle shapes. In contrast, the shear strength increases with the proportion of elongated particles correlatively with the increase of the corresponding force and fabric anisotropies. By a detailed analysis of the contact network topology, we show that various contact types contribute differently to force transmission and friction mobilization.
\end{abstract}

DOI: 10.1103/PhysRevE.94.042901

\section{INTRODUCTION}

One of the compelling properties of granular materials is that an homogenous mixture of particles is difficult to achieve when they differ in size, shape, and material properties such as density and friction [1-8]. Particle segregation occurs when a granular mixture is subjected to flow or vibration and results in more or less full "demixing" of different species [9-13]. Size segregation has been extensively studied in the past [14-18], but recently it has been shown that particle shapes also lead to segregative behavior [7,19-22]. The segregation phenomena, which represent a fundamental but often embarrassing property of granular media, are well known in particle processing industries, such as civil engineering for the elaboration of concrete and food and pharmaceutical industries for the handling of particles of different sizes. Most applications require a homogeneous product, with stringent requirements on the dispersion of constituents and the acceptable level of fluctuations. The level of segregation can affect both the local and global properties of the behavior of a granular material.

Whether numerically or by experiments, the quasistatic behavior of granular mixtures has mainly been studied for two different particle sizes [23]. The packing fraction of homogeneous mixtures of bidisperse disks is found to be higher than that of monodisperse packings, and the shear strength is maximum at intermediate mixture ratio. Very recently, it has also been evidenced that force distribution is broader in packings composed of a mixture of smooth and rigid particles than in packings composed of only rigid disks [24]. These results extend in fact those observed in the quasistatic shearing of homogeneous polydisperse packings of disks and irregular pentagons $[25,26]$.

The properties of granular materials with noncircular and aspherical shapes have only recently been investigated in a

\footnotetext{
*emilien.azema@umontpellier.fr

${ }^{\dagger}$ itthichai.p@cmu.ac.th

${ }^{\ddagger}$ fradjai@mit.edu
}

systematic fashion [27-40]. A nonlinear evolution of packing fraction and shear strength is observed when particle shape increasingly deviates from circular shape [41]. However, the geometrical and mechanical properties of mixtures of different particle shapes have not yet been investigated. Furthermore, to our best knowledge, systematics analysis has always been performed only for homogenous systems. As previously mentioned, the mixtures of different particle shapes and sizes are generally inhomogeneous and, hence, it is crucial to understand and quantify also the effects of the degree of homogeneity on the rheology. It is clear that such a control of the degree of homogeneity is quite challenging in practice and, for this reason, it has not been investigated in the past.

The aim of this paper is to systematically explore the combined effects of mixture ratio and the level of homogeneity on the mechanical behavior of sheared binary mixtures composed of elongated and circular particles by means of contact dynamics simulations. In particular, an interesting issue that we would like to address in this paper is to quantify to which extent a "good mixture" leads to "better" rheological properties. Among all possible particle shapes, elongated particles were used since their shape can be quantified by a single shape parameter representing their anisometry. Furthermore, it is well established that elongated particles tend to develop orientational order affecting fabric, force transmission, and frictional behavior as compared to circular particles [32,42-47].

In this work, packing homogeneity was represented by the Lacey mixing index $[48,49]$, which varies from zero for homogenous packing to one for fully segregated packings. We numerically construct different packings, each characterized by a mixture ratio and a Lacey mixing index. The packings are analyzed in the steady state in terms of their shear strength, packing fraction, connectivity, and fabric and force anisotropies as functions of these two parameters.

In the following, we introduce in Sec. II the numerical approach, system characteristics, and loading parameters. In Sec. III we focus on the evolution of shear strength and packing fraction with mixture parameters. The microstructure 
is analyzed in Sec. IV in terms of connectivity, and contact and force anisotropies. Section V presents concluding remarks and a summary of our main results.

\section{MODEL DESCRIPTION}

\section{A. Numerical procedures}

The simulations were carried out by means of the contact dynamics CD method [19,50-52]. The CD method is based on implicit time integration of the equations of motion and a nonsmooth formulation of mutual exclusion and dry friction between particles. This method requires no elastic repulsive potential and no smoothing of the Coulomb friction law for the determination of forces. The nonsmooth contact laws, which relate the impulsions exerted at each contact with the change of relative velocity during the time step, supposes that grains are perfectly rigid. The formulation of these contact laws involves two coefficients of restitution (normal and tangential) that control the amount of energy dissipated during collisions; in all the simulations presented in this paper the coefficients of restitution are set to zero. An iterative algorithm of solution is used, by means of which the impulsions and changes of momentum of each grain over the time step are determined. For specific implementation of the contact dynamics method see, for instance, Ref. [51].

For a systematical investigation of the effect of mixture ratio and mixture homogeneity, strict procedures need to be used in order to generate packings with continuously varying texture. The mixture ratio parameter $\alpha$ is the proportion of elongated particles. We varied $\alpha$ from 0 for a packing composed of only disks to 1 for packing composed of only elongated particles. We have $N_{p}=13000$ particles, $N_{p}^{\alpha}=\alpha N_{p}$ elongated particles and $(1-\alpha) N_{p}$ disks.

Mixture homogeneity can be measured by various mixing indices. A common definition is that of Lacey, which is directly proportional to the standard deviation from a given mean property of one of the constituents of a mixture [48,49]. Let $y_{i}$ be the $y$ coordinate of the $i$ th elongated particle. The average position of elongated particles is given by $\langle y\rangle=\left(\sum_{i=1}^{N_{p}^{\alpha}} y_{i}\right) /\left(N_{p}^{\alpha}\right)$ and their variance by $S^{2}=\left[\sum_{i=1}^{N_{p}^{\alpha}}\left(y_{i}-\right.\right.$ $\left.\langle y\rangle)^{2}\right] /\left(N_{p}^{\alpha}-1\right)$. Numerically, it is easy to construct fully segregated granular systems. We choose to place all elongated particles in a band of thickness $\epsilon$ located at the center of the packing. In the segregated case, $N_{p}^{\alpha} / n_{b}$ elongated particles are randomly distributed along $n_{b}$ horizontal bands of thickness $\delta H=H / n_{b}$, where $H$ is the initial height of the packing. From these two known states, for a given mixture ratio, the Lacey parameter $M$ is given by

$$
M=\frac{S_{r}^{2}-S^{2}}{S_{r}^{2}-S_{0}^{2}},
$$

where $S_{r}$ and $S_{0}$ are the values of the variance calculated from the homogenous and fully segregated systems, respectively. Hence $M$ varies from 0 , for fully segregated system, to 1 , for fully homogeneous mixture.

The issue is to efficiently distribute elongated particles inside the material in order to obtain the desired value of $M$, with a continuous transition from fully segregated to fully homogeneous state initially constructed. Thus, given Eq. (1)

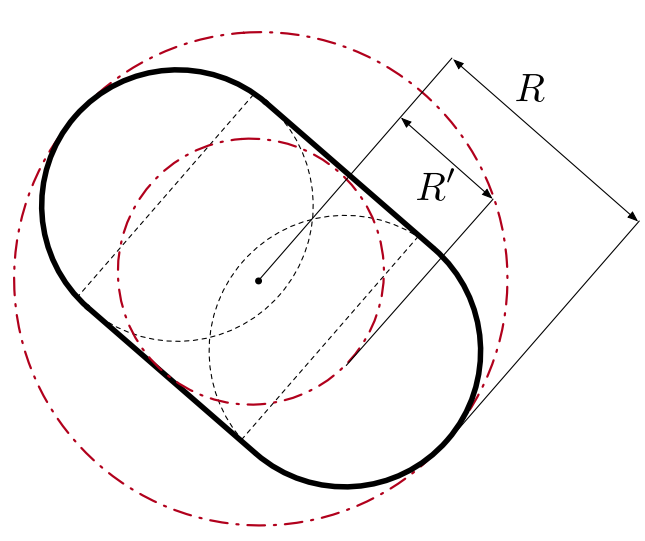

FIG. 1. Geometry of rcr particles.

and the way fully segregated and homogenous states are built, we randomly distribute the elongated particles in subband of thickness $\delta H^{\prime}=H^{\prime} / n_{b}$, where $H^{\prime}$ is the thickness of the central band defined by

$$
\frac{H-H^{\prime}}{H-\epsilon}=M^{*},
$$

where $M^{*}$ represents the homogeneity parameter. At the end of the construction, the Lacey parameter $M$ is computed and compared to the desired homogeneity parameter $M^{*}$. Due to the inherent disorder of granular media, we do not expect to obtain the exact imposed value. In practice, our mixtures are defined with a precision of $\left|M-M^{*}\right|<0.01$.

\section{B. Elongated particles}

The elongated particles are modeled as a juxtaposition of two half-disks of radius $R^{\prime}$ and one rectangle of length $L$ and width $2 R^{\prime}$ [32] as shown in Fig. 1 . In the following, we will refer to these particles as "rounded-cap rectangles" (rcr). The elongation of rcr particles is defined by the dimensionless parameter $\eta$ [41], or the aspect ratio $\lambda$ as follows:

$$
\eta=\frac{\Delta R}{R}=\frac{\lambda-1}{\lambda}
$$

where $R=L / 2+R^{\prime}$ is the radius of the circle circumscribing the particle, $\Delta R=R-R^{\prime}=L / 2$ and $\lambda=(L+2 R) /(2 R)$. $\eta$ varies from 0 for a circle to 1 , corresponding to a line of zero thickness. As it was reported by a number of studies, particle shape elongation affects drastically the rheological properties of granular media. For instance, a well-known result is that the packing fraction varies unmonotonically as particles become more elongated [28,29,53,54], whereas the shear strength increases linearly [32]. In our simulations $\eta$ is fixed to be 0.7 for which we know that in the critical state (see below), the packing fraction of packing composed with only rcr particles nearly coincides with that of packings of disks, whereas the shear strength is $40 \%$ higher $^{1}[32,55]$.

\footnotetext{
${ }^{1}$ Note that a series of simulations were also performed with $\eta=0.4$, for which we know that the packing fraction of packing composed with only rcr particles is maximal. In this case the shear strength is slightly larger than that of a system composed of only disks, but the trends that are presented later are basically the same.
} 


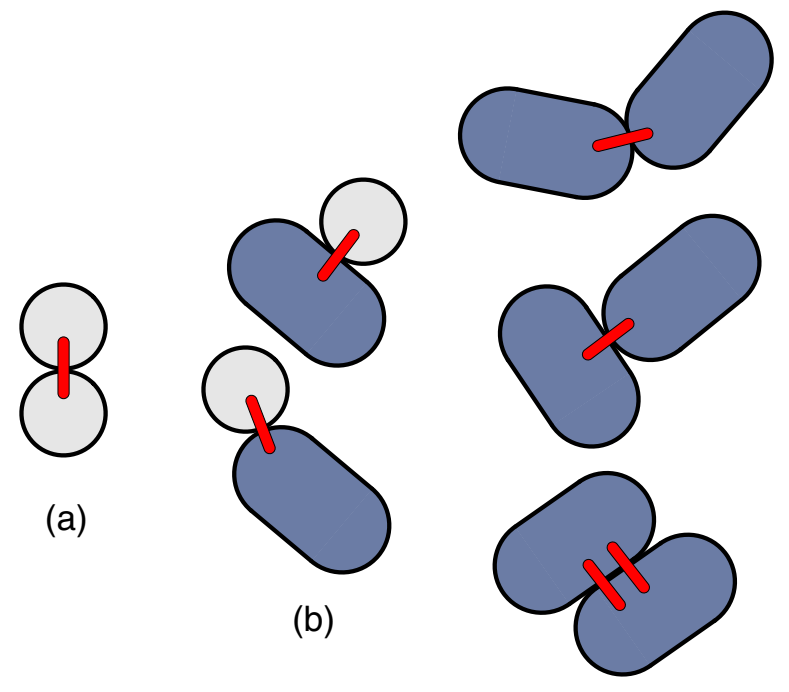

(c)

FIG. 2. Representations of disk-disk (dd), disk-rcr (dr), and rerrcr (rr) contacts.

For a mixture of disks and rer particles three kinds of contacts may arise: disk-disk, disk-rcr, and rcr-rcr. On the other hand, since the rcr particles are modeled as clusters of two disks and one rectangle, the disk-rcr contacts may occur in the form of disk-disk or disk-side contacts, and rer-rcr contacts in the form of disk-disk, disk-side, or side-side contacts. In this last case, two contact points are necessary to represent side-side contacts, as shown in Fig. 2. The procedure of contact detection between polygonal particles and its implementation in the framework of the CD method are described in detail in Ref. [36].

\section{Sample construction and biaxial test}

All samples are prepared according to the same protocol. First, a dense packing composed of 13000 disks is constructed by means of a layer-by-layer deposition model based on simple geometrical rules [56]. The particles are deposited sequentially on a substrate. Each new particle is placed at the lowest possible position at the free surface as a function of its diameter. This procedure leads to a dense packing in which each disk is supported by two underlying particles and supports one or two other particles. In order to avoid long-range ordering, a weak size polydispersity is considered by varying the diameter $d$ of the disks in the range of $\left[d_{\min }, 2 d_{\min }\right]$ with a uniform distribution of particle volume fractions.

For each choice of the parameters $\alpha$ and $M^{*}$, and according to the procedure established above (see Sec. II A), $\alpha N_{p}$ disks are replaced by rcr particles with each disk serving as the circumscribing circle of a rer particle. Note that the rcr particles are introduced with random orientation in the disk.

Following this geometrical process, the packing is compacted by isotropic compression inside a rectangular frame of dimensions $l_{0} \times h_{0}$ in which the left and bottom walls are fixed, and the right and top walls are subjected to the same compressive stress $\sigma_{0}$. The gravity $g$ and friction coefficients $\mu$ between particles and with the walls are set to zero during

the isotropic compression in order to avoid force gradients and obtain isotropic dense packings. This procedure is stopped when a persistent contact force network is obtained and fluctuations around the mean values of the solid fraction and connectivity of the contact network remain below $0.1 \%$. Figure 3 displays snapshots of the packings for several values of $M^{*}$ and $\alpha$ at the end of isotropic compaction.

The isotropic samples are then subjected to vertical compression by downward displacement of the top wall at a constant velocity $v_{y}$ for a constant confining stress $\sigma_{0}$ acting on the lateral walls. The friction coefficient $\mu$ between particles is set to 0.5 and to zero with the walls. Since we are interested in quasistatic behavior, the shear rate should be such that the kinetic energy supplied by shearing is negligible compared to the static pressure. This can be formulated in terms of an inertia parameter I defined by [57]

$$
I=\dot{\varepsilon} \sqrt{\frac{m}{p}},
$$

where $\dot{\varepsilon}=\dot{y} / y$ is the strain rate, $m$ is the particle mass, and $p$ is the mean pressure. The quasistatic limit is characterized by the condition $I \ll 1$. In our simulations, $I$ was below $10^{-3}$.

We performed a large number of biaxial simulations for a broad set of combinations of the mixture ratio and homogeneity parameters. The mixture ratio $\alpha$ was varied from 0.1 to 0.9 by steps of 0.1 , and, for each value of $\alpha$, the homogeneity parameter was varied in the set $\{0.0,0.1,0.2, \ldots, 0.8,0.9,0.97\}$. Two other biaxial tests were performed for packings composed of only disks $(\alpha=0)$ and rcr particles $(\alpha=1)$. Hence a total number of two times 110 simulations were performed: (1) isotropic compression and (2) biaxial test from the isotropic state constructed. For video samples of the simulations, see www.cgp-gateway.org/ref036.

\section{MACROSCOPIC BEHAVIOR}

The shear strength of granular materials is characterized by the coefficient of internal friction $\varphi$, which can be calculated from the stress tensor $\sigma$ defined by $[19,58]$

$$
\sigma_{i j}=\frac{1}{V} \sum_{c \in V} f_{i}^{c} \ell_{j}^{c}
$$

where $\ell_{j}^{c}$ is the $j$ component of the branch vector joining the centers of the two touching particles at contact $c, f_{i}^{c}$ is the $i$ component of the force vector at contact $c$, and $V$ is the total volume. The macroscopic friction coefficient is given by $\mu=\sin \varphi=q / p$, where $p=\left(\sigma_{1}+\sigma_{2}\right) / 2$ is the mean stress, $q=\left(\sigma_{1}-\sigma_{2}\right) / 2$ is the stress deviator, and $\sigma_{1}$ and $\sigma_{2}$ are the principal stresses. For biaxial shearing, the major principal direction during compression is assumed to be vertical.

Figure 4 shows the normalized shear stress $q / p$ and packing fraction $\rho$ as a function of shear strain $\varepsilon_{q}$ for five extreme combinations (see inset) of the mixture ratio $\alpha$ and mixture homogeneity $M^{*}$. The shear strain $\varepsilon_{q}$ is evaluated from the difference between the vertical and horizontal strain defined respectively by $\varepsilon_{1}=\int_{h_{0}}^{h} \frac{d h^{\prime}}{h^{\prime}}=\ln \left(1+\frac{\Delta h}{h_{0}}\right)$ and $\varepsilon_{2}=\int_{l_{0}}^{l} \frac{d l^{\prime}}{l^{\prime}}=$ $\ln \left(1+\frac{\Delta l}{l_{0}}\right)$, where $l_{0}$ and $h_{0}$ are the initial box width and height. $\Delta h=h-h_{0}$ is the total downward displacement, and $\Delta l=l-l_{0}$ is the total change of the box width. 


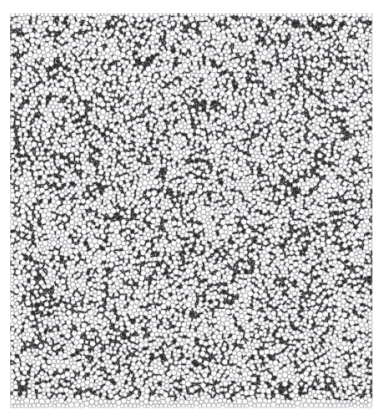

(a)
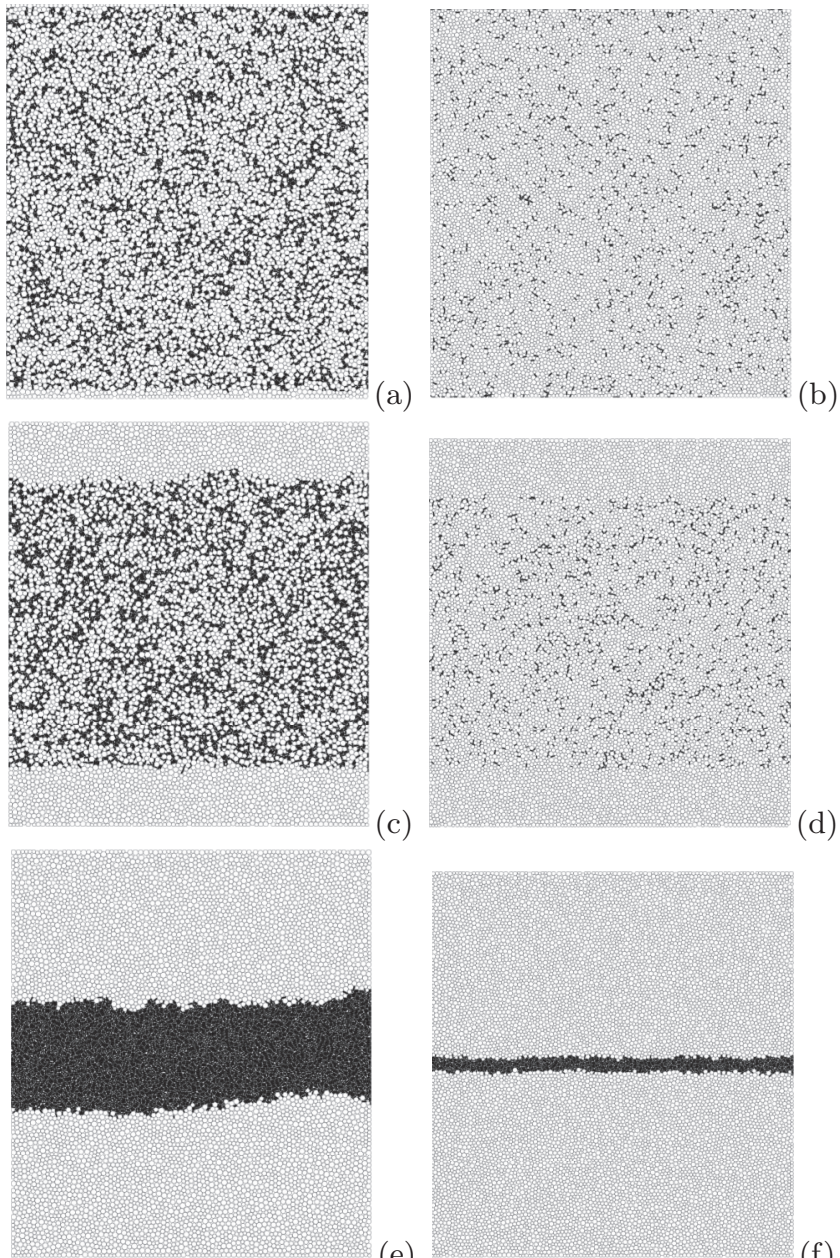

(e)
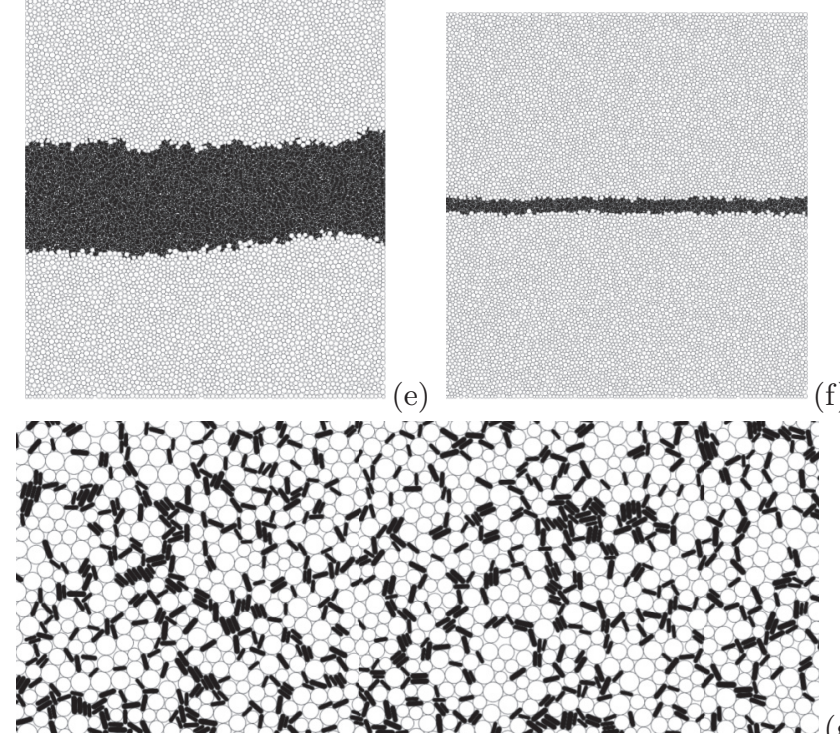

$(\mathrm{g})$

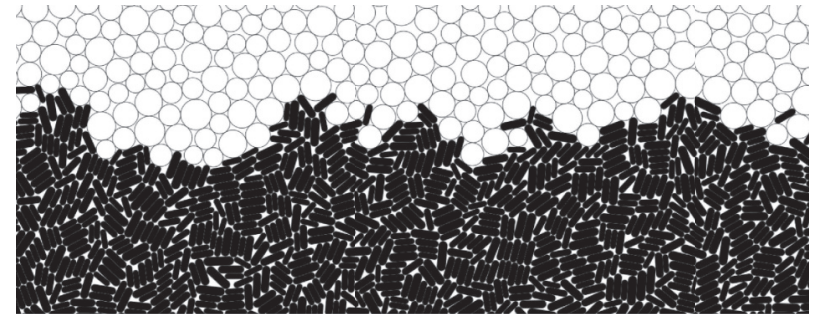

(h)

FIG. 3. Examples of the generated packings at the initial state, for $\alpha=0.5$ (left column) and $\alpha=0.1$ (right column) and for $M^{*}=$ 0.0 (a),(b), $M^{*}=0.5$ (c),(d), and $M^{*}=1.0$ (e),(f). A zoom near the central zone is shown for $\alpha=0.5$ and $M^{*}=0.0(\mathrm{~g})$ and $M^{*}=$ 1.0 (h).

During biaxial compression, the shear stress increases initially to a high value before decreasing to a constant value in the steady state. The jump observed at $\varepsilon_{q}=0$ reflects both the rigidity of the particles and high initial packing fraction

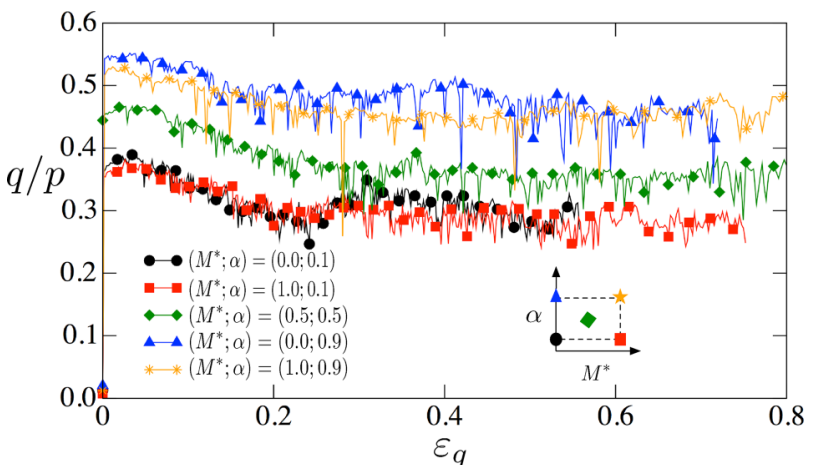

(a)

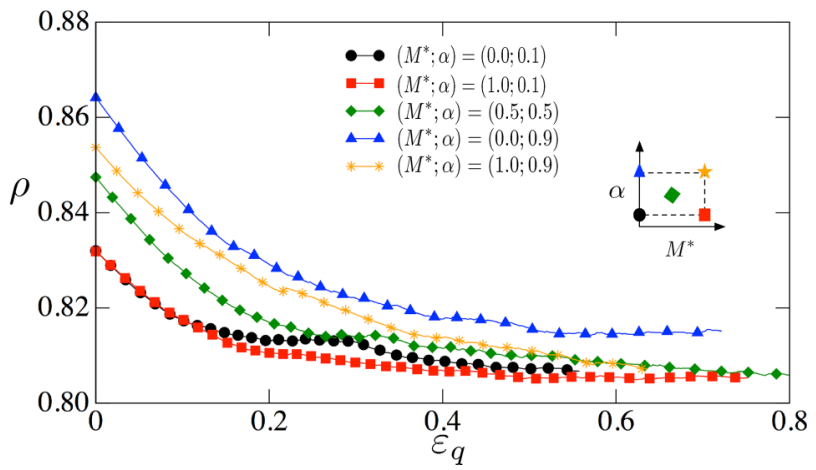

(b)

FIG. 4. Normalized shear stress $q / p$ (a) and packing fraction $\rho$ (b) as a function of cumulative shear strain $\varepsilon_{q}$ for five extreme combinations (see schematic representation in inset) of the mixture ratio $\alpha$ and mixture homogeneity $M^{*}$.

of the samples induced by initial isotropic compaction. All samples dilate during shear and $\rho$ declines from its value $\rho^{\text {iso }}$ (see Sec. II) in the initial isotropic state and levels off at a constant value $\rho^{*}$ in the steady state. The samples undergo an almost homogeneous dilation at low shear strains and thus $\rho$ decreases rapidly. At larger strains, a nearly homogeneous packing fraction $\rho^{*}$ is reached practically at $\varepsilon_{q}=0.4$ for all samples. Both $\rho^{*}$ and the friction coefficient $\sin \varphi^{*}$, defined from the mean value of $q / p$ in the steady state, are independent of the initial state, and thus for our rigid particles $\rho^{*}$ and $\varphi^{*}$ represent intrinsic properties of the material at the macroscopic scale [59].

Figure 5 shows the average values of $\sin \varphi^{*}$ as a function of both $\alpha$ and $M^{*}$. As expected, the shear strength increases as the proportion $\alpha$ of elongated particles increases. We note also that this increase is nearly linear for all values of $M^{*}$. We also observe that, for a given value of $\alpha$, the shear strength is practically independent of $M^{*}$. This finding is a rather counterintuitive finding as one should rather expect a decrease of the strength for $M^{*} \rightarrow 1[60,61]$. In particular, knowing the ability of elongated particles to orient themselves perpendicular to shear direction (see [32,42,43,45-47], and Sec. IV), a "naive" picture is that segregation should create a smooth and rigid surface enhancing the sliding of disk over the set of elongated particles at their interface. We will see in Sec. IV C that this idea is partially true.

We also observe in Fig. 6 that the packing fraction is nearly independent of both $\alpha$ and $M^{*}$. The independence of $\rho^{*}$ with respect to $\alpha$ was expected only for $M^{*} \rightarrow 1$. Indeed, as mentioned in Sec. II B, systems composed of only rcr particles 

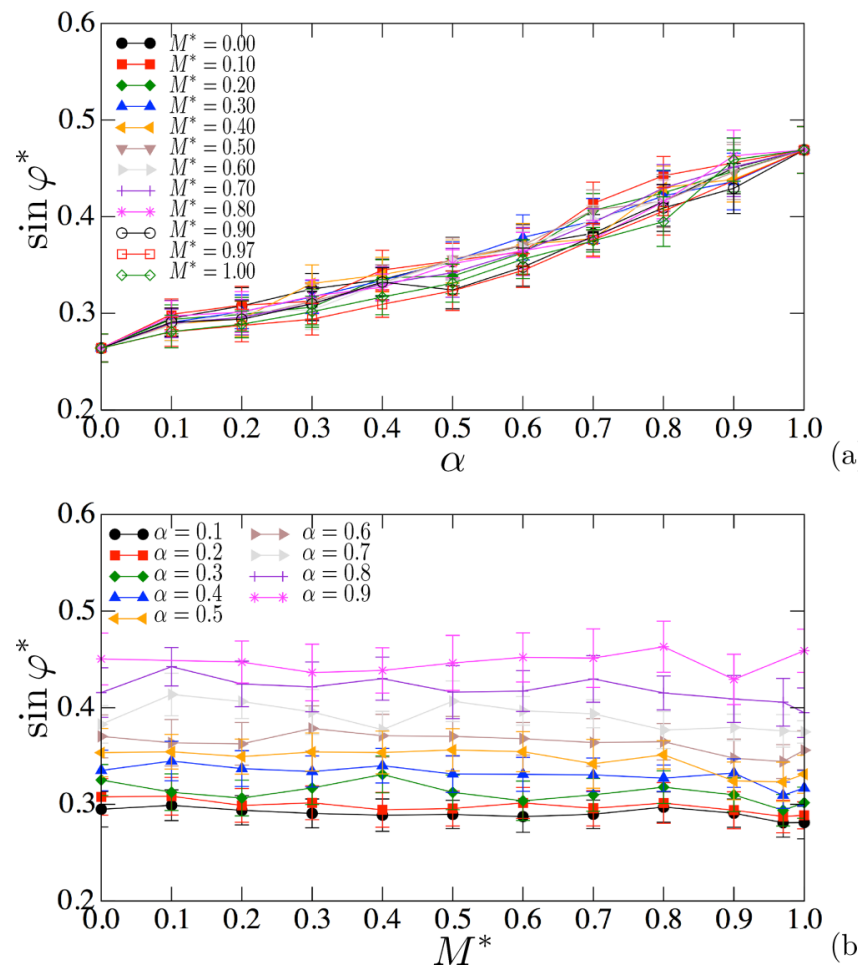

(b)

FIG. 5. Macroscopic friction angle $\sin \varphi^{*}$ as a function of the ratio (a) and homogeneity (b) of the mixture for all values of $M^{*}$ (a) and $\alpha$ (b) averaged in the steady state. Error bars correspond to the standard deviation of the fluctuations in the steady state.

with elongation parameter $\eta=0.7$ have nearly the same packing fraction as the disk packing. ${ }^{2}$ This is again a surprising result that the packing fraction is only very weakly dependent on the value of $\alpha$. This is also a rather counterintuitive behavior as it is often believed that the shear strength in granular materials should increase with solid fraction.

\section{GRANULAR TEXTURE, FORCE TRANSMISSION, AND FRICTION MOBILIZATION}

A well-known feature of dry granular materials is that the shear strength results from the buildup of various anisotropic structures during shear due to (i) friction between the particles and (ii) steric effects depending on particle shapes and size distributions [62-66]. Figure 7 shows a typical map of normal forces $f_{n}$ represented by the thickness of the lines joining particle centers to the contact points, and by friction mobilization at contact $c$ represented by circles whose diameters are proportional to the mobilization index $I_{m}^{c}=\left|f_{t}^{c}\right| /\left(\mu f_{n}^{c}\right)$ [35], where $f_{t}^{c}$ is the tangential force. Visual inspection reveals the anisotropic and inhomogenous nature of the contact and forces networks. In particular, these features are enhanced when packings tend to be segregated. Indeed, for fully segregated packings [here $M^{*}=1$; Fig. 7(b)], the mobilized contacts are

\footnotetext{
${ }^{2}$ Note that the packing fraction increases with $\alpha$ in the case of rcr particles with elongation parameter $\eta=0.4$ but, again, the shear strength and packing fraction remains also nearly independent with $M^{*}$.
}

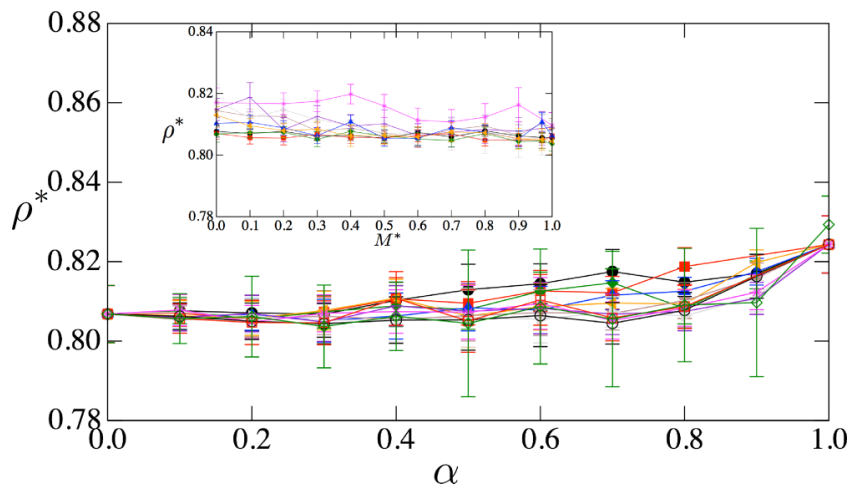

FIG. 6. Packing fraction $\rho^{*}$ as a function of the proportion $\alpha$ of rcr particles for all values of $M^{*}$ averaged in the residual state. Inset: $\rho^{*}$ as a function of $M^{*}$ for all values of $\alpha$. Legend are the same as for Figs. 5(a) and 5(b) for the inset. Error bars correspond to the standard deviation of the fluctuations in the steady state.

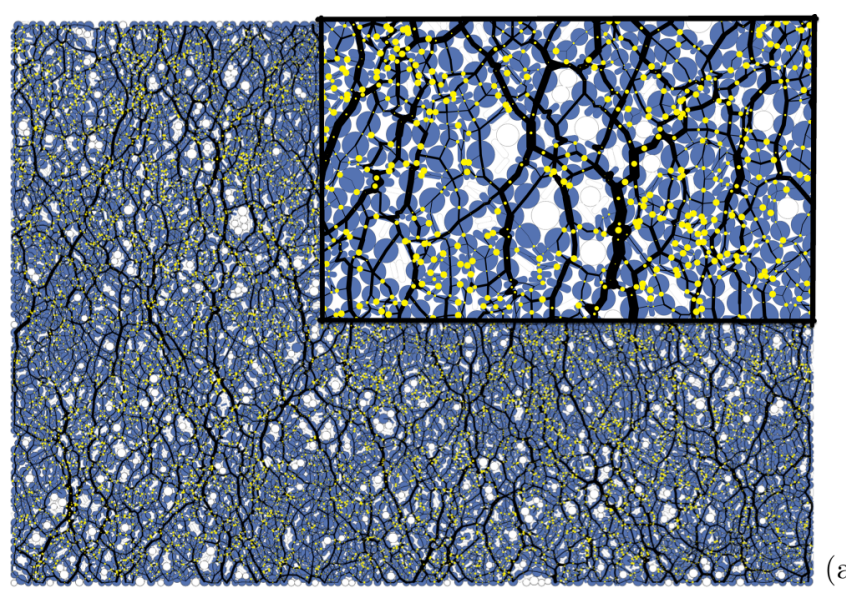

(a)

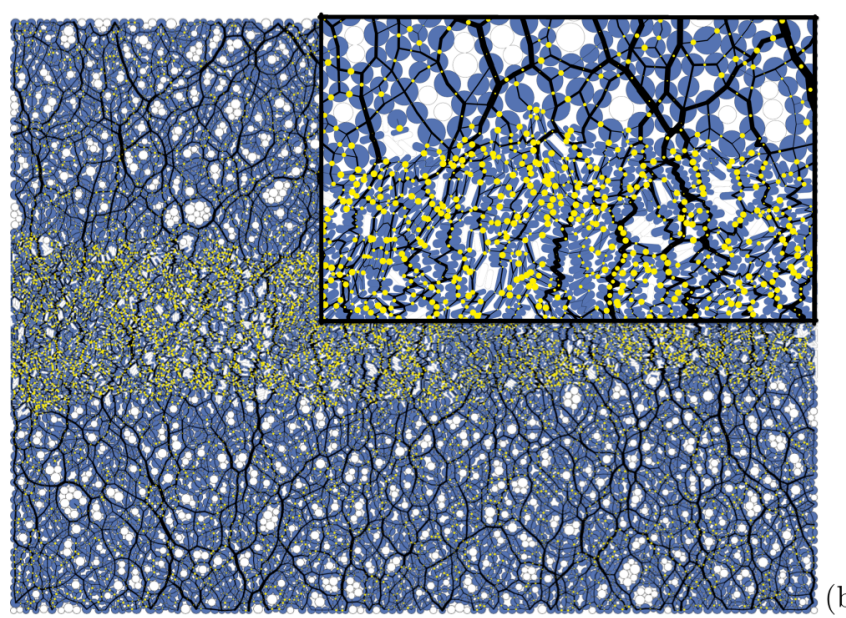

FIG. 7. Snapshots of force-bearing particles for (a) $\left(\alpha, M^{*}\right)=$ $(0.50,0.00)$ and (b) $\left(\alpha, M^{*}\right)=(0.5,1.00)$ in the critical state at $\varepsilon_{q} \simeq$ 0.5 . Floating particles (i.e., particles with one or no contacts) are shown in white and normal forces are represented by the thickness of the segments joining the particle centers to the contact point. The diameters of light-gray (yellow) circles are proportional to the friction mobilization $I_{c}=\left|f_{t}^{c}\right| /\left(\mu f_{n}^{c}\right)$ at each contact $c$. 
mainly concentrated in the central part of the packing with contacts between rcr-rcr particles for which normal forces have a marked "zigzag" shape. In contrast, for fully homogenous systems [here $M^{*}=0$; Fig. 7(a)] no peculiar organization of normal forces or mobilized contacts is observed.

Below, we investigate the general organization (texture) of our sheared packings in terms of particle connectivity, and force and fabric anisotropies of the contact network. First, we briefly present the statistical descriptors of the granular microstructure and force transmission, in terms of density and average force and branch vector length as a function of contact orientation.

\section{A. Stress partition}

Since the shear stress corresponds to the deviation of stress components from the mean stress $p$ along different space directions, the most useful information encoded in the microstructure is the contact density and average normal and tangential forces as a function of contact orientations. Let us introduce the probability distribution function $P(\boldsymbol{n})$ of contact orientations $\boldsymbol{n}$ and the average branch vector $\langle\boldsymbol{\ell}\rangle(\boldsymbol{n})$ as a function of $\boldsymbol{n}$ as a descriptors of the anisotropies of the contact network. Likewise, the force anisotropy can be characterized by the average contact force $\langle\boldsymbol{f}\rangle(\boldsymbol{n})$ as a function of $\boldsymbol{n}$. In two dimensions, the unit vector $\boldsymbol{n}$ is parametrized by a single angle $\theta$, and the branch vector $\ell$ and contact force $f$ can be represented by their normal and tangential components: $\ell=$ $\ell_{n} \boldsymbol{n}+\ell_{t} \boldsymbol{t}$ and $\boldsymbol{f}=f_{n} \boldsymbol{n}+f_{t} \boldsymbol{t}$. Thus the probability density $P(\theta)$ of contact orientations $\theta$, the angular averages of the components $\left\langle\ell_{n}\right\rangle(\theta)$ and $\left\langle\ell_{t}\right\rangle(\theta)$ of the branch vectors and the average components $\left\langle f_{n}\right\rangle(\theta)$ and $\left\langle f_{t}\right\rangle(\theta)$ of forces as a function of contact orientation $\theta$ provide a rich description of the anisotropic state of a granular material [26,35,38,39,62,67,68].

As shown in Fig. 8, for sheared systems the above functions tend to take a simple unimodal shape, which can be well approximated by their truncated Fourier expansions:

$$
\begin{aligned}
P(\theta) & =\frac{1}{2 \pi}\left\{1+a_{c} \cos 2\left(\theta-\theta_{c}\right)\right\} & & (a), \\
\left\langle\ell_{n}\right\rangle(\theta) & =\left\langle\ell_{n}\right\rangle\left\{1+a_{l n} \cos 2\left(\theta-\theta_{l n}\right)\right\} & & (b), \\
\left\langle\ell_{t}\right\rangle(\theta) & =\left\langle\ell_{n}\right\rangle a_{l t} \sin 2\left(\theta-\theta_{l t}\right) & & (c), \\
\left\langle f_{n}\right\rangle(\theta) & =\left\langle f_{n}\right\rangle\left\{1+a_{f n} \cos 2\left(\theta-\theta_{f n}\right)\right\} & & (d), \\
\left\langle f_{t}\right\rangle(\theta) & =\left\langle f_{n}\right\rangle a_{f t} \sin 2\left(\theta-\theta_{f t}\right) & & (e),
\end{aligned}
$$

where $a_{c}$ is contact orientation anisotropy, $a_{l n}$ is normal branch anisotropy, $a_{l t}$ is tangential branch anisotropy, $a_{f n}$ is normal force anisotropy, and $a_{f t}$ is tangential force anisotropy. The angles $\theta_{c}, \theta_{l n}, \theta_{l t}, \theta_{f n}$, and $\theta_{f t}$ are the corresponding privileged directions. In practice, anisotropic parameters are calculated through the force and fabric tensors presented in the Appendix. The analytical forms of $\left\langle\ell_{t}\right\rangle$ and $\left\langle f_{t}\right\rangle$ result from the orthonormal nature of the Fourier basis and the fact that the mean value of $\ell_{t}$ and $f_{t}$ vanish as a consequence of, respectively, axial symmetries and force balance:

$$
\int_{0}^{\pi}\left\langle\ell_{t}\right\rangle(\theta) P(\theta) d \theta=\int_{0}^{\pi}\left\langle f_{t}\right\rangle(\theta) P(\theta) d \theta=0 .
$$

The anisotropies $a_{c}, a_{l n}, a_{l t}, a_{f n}$, and $a_{f t}$ are interesting not only as descriptors of the granular microstructure and force transmission, but also it can be shown that the general
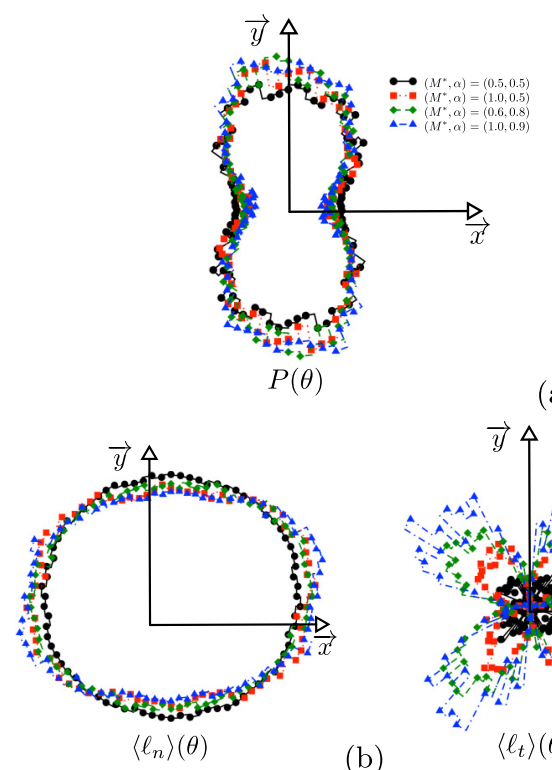

(a)

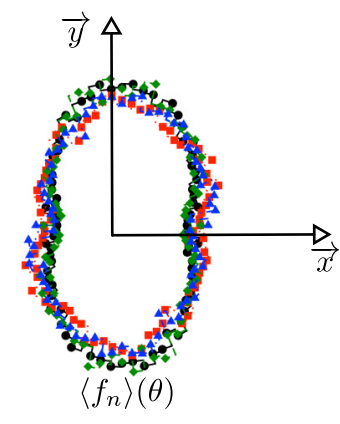

(b)

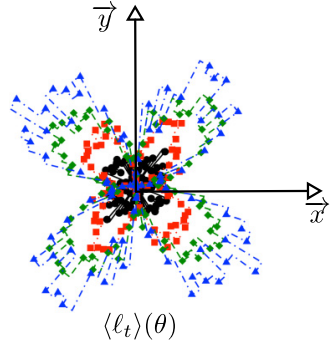

(c)

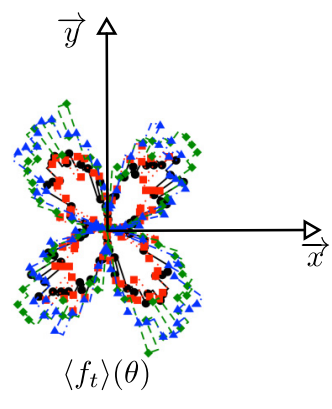

(e)

FIG. 8. Polar representation of the functions $P(\theta)(\mathrm{a}),\left\langle\ell_{n}\right\rangle(\theta)(\mathrm{b})$, $\left\langle\ell_{t}\right\rangle(\theta)(\mathrm{c}),\left\langle f_{n}\right\rangle(\theta)(\mathrm{d})$, and $\left\langle f_{t}\right\rangle(\theta)$ (e) for different values of the $M^{*}$ and $\alpha$.

expression of the stress tensor (5) leads to the following simple relation $[32,39,67,69]$ :

$$
\sin \varphi^{*} \simeq \frac{1}{2}\left(a_{c}+a_{l n}+a_{l t}+a_{f n}+a_{f t}\right),
$$

where the cross products between the anisotropy parameters have been neglected.

In the following section, we analyze the anisotropies and their respective roles in the shear strength as a function of the degree of homogeneity and mixture ratio.

\section{B. Anisotropy of the contact network and forces}

Figures 9 and 10 show the variations of the contact anisotropy and the normal and tangential branch anisotropies, $a_{c}, a_{\ell n}$, and $a_{\ell t}$, respectively, averaged in the steady state, as a function of the proportion $\alpha$ of elongated particles for all values of $M^{*}$ and as a function of $M^{*}$ for all values of $\alpha$. We see that $a_{c}$ is positive and increases with $\alpha$. Both $a_{\ell n}$ and $a_{\ell t}$ are small compared to $a_{c}$ but they are negative and they increase in absolute value with $\alpha$. A negative value means that, on average, the normal and tangential components of branch vectors are preferentially perpendicular to the major principal stress direction.

These variations of the geometrical anisotropies with $\alpha$ are compatible with the well-known trend of elongated 

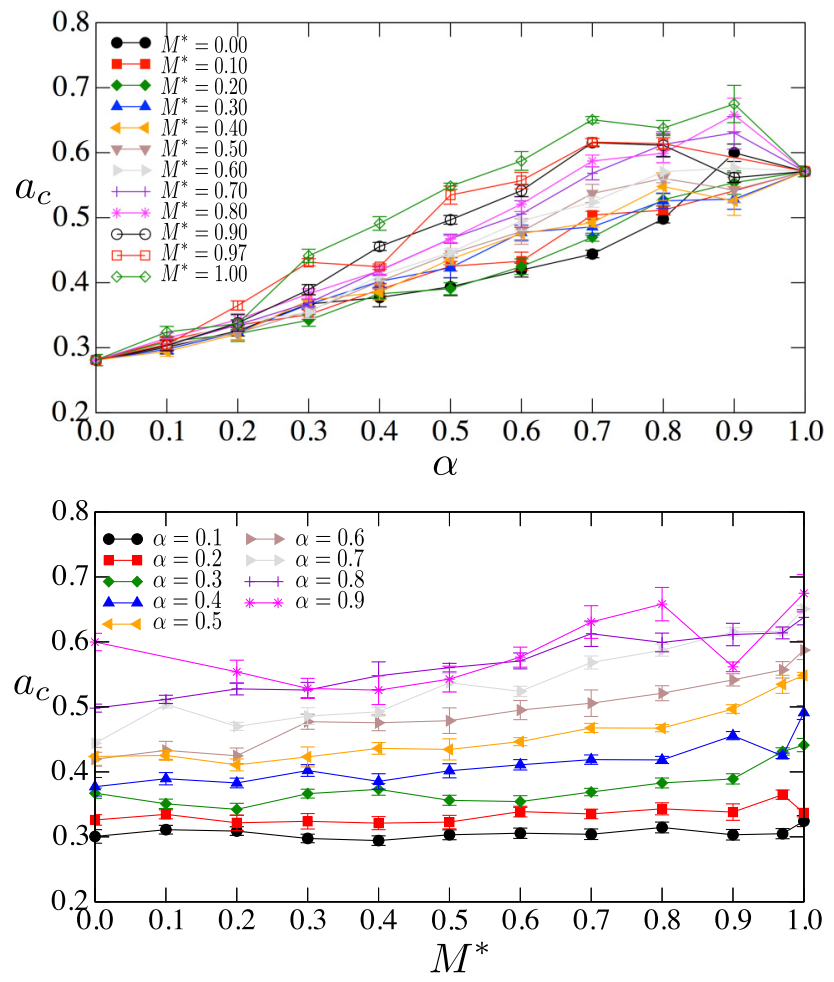

(a)

FIG. 9. Evolution of contact anisotropies as a function of $\alpha$ (a) for all values of $M^{*}$ and as a function of $M^{*}$ for all values of $\alpha$ in the steady shear state. Error bars correspond to the standard deviation of the fluctuations in the steady state.

particles to orient themselves perpendicularly to the shear direction [32,42,43,45-47], sandwiched between two disks at low values of $M^{*}$ [see snapshot in Fig. 7(a)] or in the form of columnar structures when $M^{*} \rightarrow 1$ [see snapshot of Fig. 7(b)].

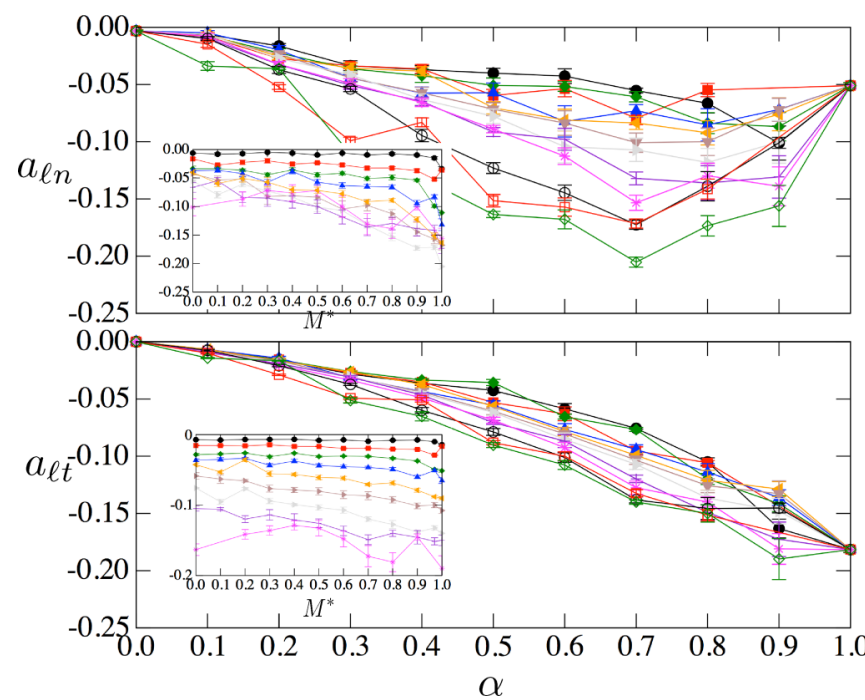

FIG. 10. Evolution of normal (up) and tangential (down) branch anisotropies as a function of $\alpha$ for all values of $M^{*}$ and in the insets as a function of $M^{*}$ for all values of $\alpha$ in the steady shear state. Error bars correspond to the standard deviation of the fluctuations in the steady state. Legends are the same as in Figs. 9(a) and 9(b) for the insets.

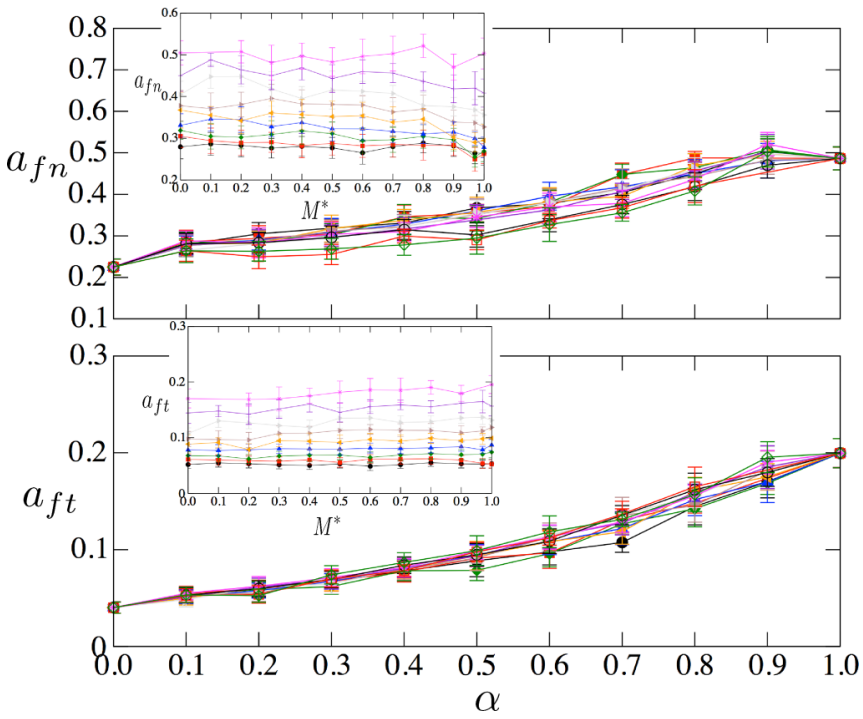

FIG. 11. Evolution of normal (up) and tangential (down) force anisotropies as a function of $\alpha$ for all values of $M^{*}$ and in the insets as a function of $M^{*}$ for all values of $\alpha$ in the steady shear state. Error bars correspond to the standard deviation of the fluctuations in the steady state. Legends are the same as for Figs. 9(a) and 9(b) for the insets.

In all cases, particles form longer branch vectors with their neighbors in the direction of extension, which suggest that the particles touch preferentially along their minor axes when the contact orientation is close to the compression axis, and along their major axis when the contact orientation is close to the extension axis.

In contrast, we see that all geometrical anisotropies are nearly independent with the level of homogeneity, except maybe for $\alpha=0.6$ and 0.7 for which we can detect a slight increase of $a_{c}$ and $\left|a_{\ell n}\right|$ from 0.42 to $\simeq 0.62$ and from 0.05 to 0.18 , respectively. As it was the case for the macroscopic response, this observation is again very surprising because visual inspection in Figs. 3 and 7 illustrate that the structure of the packings clearly depends on the values of $M^{*}$, and one would again expect that the columnar structure developed for $M^{*} \rightarrow$ 1 would imply an increase of the geometrical anisotropies. In other words, the anisotropic parameters introduced above cannot capture the geometrical differences induced by segregation, and a specific traitement must be done (see Sec. IV C).

Figure 11 shows the variations of the normal and tangential force anisotropies, respectively $a_{f n}$ and $a_{f t}$, averaged in the steady state, as a function of $\alpha$ for all values of $M^{*}$ and as a function of $M^{*}$ for all values of $\alpha$ (inset). We see that both anisotropies increase with $\alpha$. In connection with the variations of $a_{c}$ with $\alpha$, the increase of $a_{f n}$ shows that stronger force chains are transmitted along the principal stress direction and thus the mean normal force, given by $\left\langle f_{n}\right\rangle=\int_{0}^{\pi} P(\theta)\left\langle f_{n}\right\rangle(\theta) d \theta$, increases too. In the same time, the increase of $a_{f t}$ with $\alpha$ reveals that an increasing number of contacts are mobilized in friction when disks are replaced by rcr particles. Indeed, by integrating Eq. 6(e) in the range $[0, \pi / 2]$, the mean mobilization index $\left\langle I_{\mu}\right\rangle=\left\langle\left|f_{t}\right|\right\rangle /\left(\mu\left\langle f_{n}\right\rangle\right)$ is equal to $2 a_{f t}$. This variation of $a_{f t}$ is also compatible with the 
well-known aptitude of faceted particles to mobilize friction at side-side contacts [32,36,39,70,71].

In contrast, we note again that both $a_{f n}$ and $a_{f t}$ remain constant with $M^{*}$. In other words, normal and tangential force anisotropies cannot capture the specificities of the mixture such as the peculiar distribution of mobilized contacts illustrated in Fig. 7 for segregated systems. Finally, it is also worth mentioning that both tangential force anisotropy and the absolute value of branch anisotropies increase with $\alpha$ in the same range from 0.0 to 0.2 , so that $a_{f t}+a_{\ell n}+a_{\ell t} \simeq 0$.

Thus, by virtue of Eq. (8), the increase of the macroscopic friction angle with the mixture ratio $\alpha$ is essentially due to the increase of $a_{c}$ and $a_{f n}$. In contrast, the independence of $\sin \varphi^{*}$ with respect to $M^{*}$ results from the fact that all anisotropies are also independent with $M^{*}$. This last finding is very surprising in particular when we refer to the maps of Figs. 3 and 7, in which just a visual inspection reveals profound change in the granular structures and forces. This point is discussed in much more detail in the following section.

\section{Partition of the contact network}

As mentioned in the preceding section, a distinct feature of a binary granular mixture is the possibility of forming different types of contacts. We can distinguish contacts in each set of particles having the same shape, i.e., disk-disk and rcr-rcr contacts, and contacts at the interface between each species, i.e., disk-rcr contacts. Furthermore, rcr-rcr or disk-rcr contacts may occur in the form of side-side or side-disk contacts, which are able to accommodate force lines that are usually unsustainable by disk-disk contacts. For this reason, it is worthwhile trying to isolate their respective roles with respect to the shear strength as well as the structural and forces anisotropies.

As we have seen previously that the effect of $\alpha$ results in a "simple" shift of the data, and also because we must go down to a lower scale of description to analyze precisely the effect of $M^{*}$, in this section, we consider only packing composed by the same proportion of disk and rer (i.e., $\alpha=0.5$ ).

A tricolor map of normal contact forces projected along the vectors joining the particle centers to the contact points and representing disk-disk $(d d)$, disk-rer $(d r)$, and rer-rer $(r r)$ contacts is displayed in Fig. 12, for $\alpha=0.5$ and three different values of $M^{*}$. We see here that the force chains reflect the mixing different contact types. In the homogeneous case, the spatial organization of contacts is also very homogeneous and the contact and force networks are composed of small sets of short chains composed of only disk-disk and rcr-rcr contacts, partially mediated by disk-rcr contacts. In contrast, in the fully segregated case we observe long force chains for each subset of contacts. In particular, force chains composed of only rer-rcr contacts have a marked zigzag aspect, in comparison to that of only disk-disk contacts.

\section{Partial anisotropies}

In the residual state, the proportions of different contact types are nearly constant. Figure 13 shows the proportions $k_{d d}, k_{d r}$, and $k_{r r}$ of $d d, d r$, and $r r$ contacts averaged over the
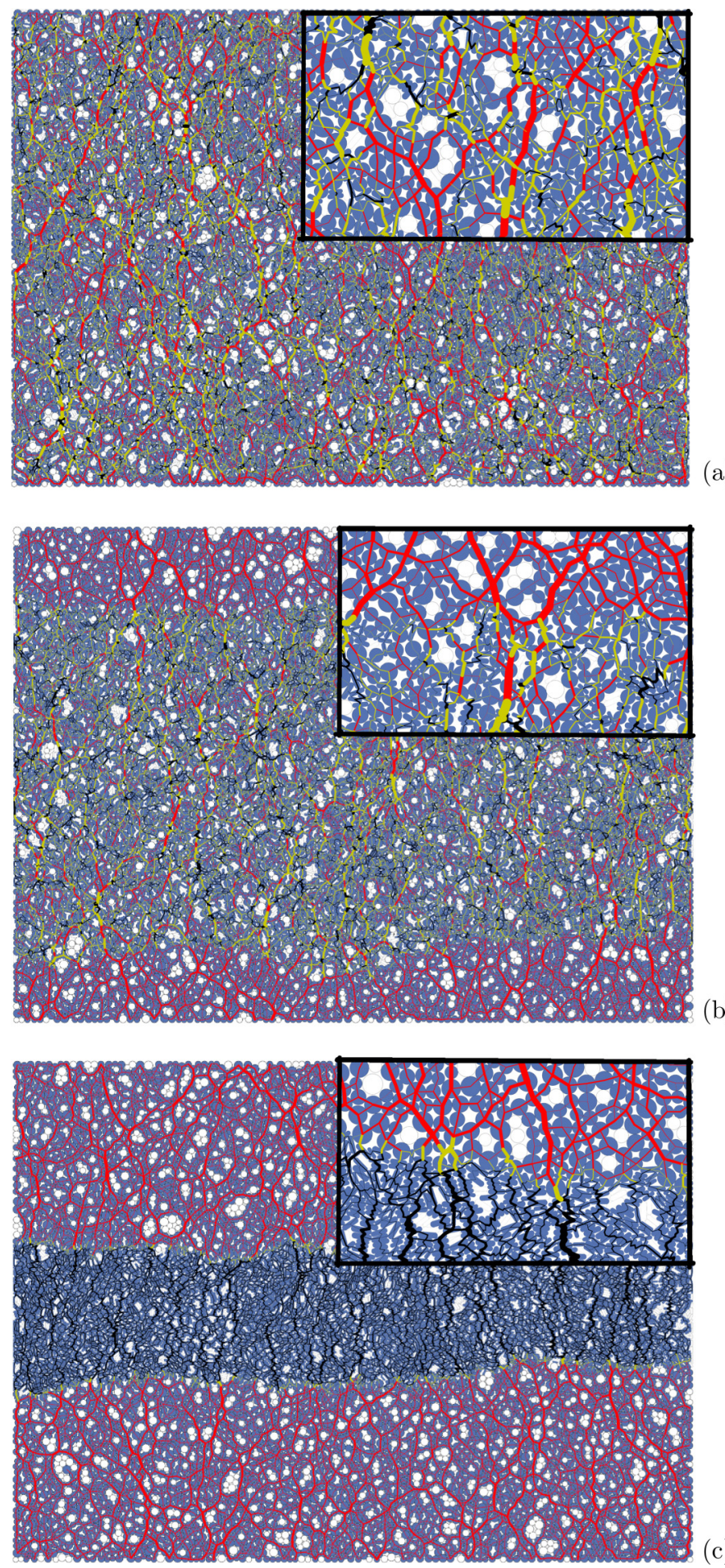

(c)

FIG. 12. Snapshots of force-bearing particles for $\alpha=0.5$ and $M^{*}=0.0(\mathrm{a}), M^{*}=0.5(\mathrm{~b})$, and $M^{*}=1.0$ (c) in the critical state at $\varepsilon_{q} \simeq 0.5$. The floating particles (i.e., particles with one or no contacts) are shown in white, and the normal forces are represented by the thickness of the segments joining the particle centers to the contact points, in red for disk-disk contacts, yellow for disk-rcr contacts, and in black for rer-rcr contacts.

residual state as a function of $M^{*}$, and for $\alpha=0.5$. We see that $k_{d r}$ declines with $M^{*}$ from 0.5 (for homogeneous packings) to $\simeq 0$ for fully segregated packings, the only $d r$ contacts being 


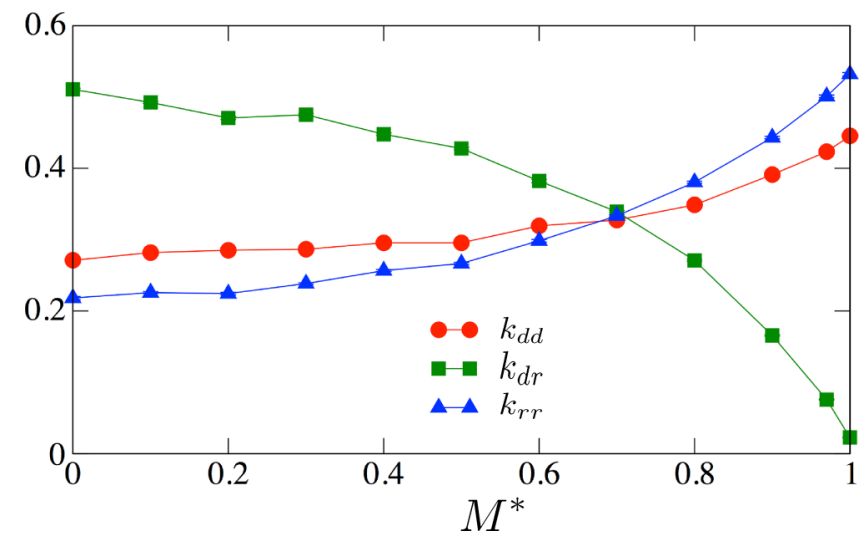

FIG. 13. Proportions of disk-disk $(d d)$, disk-rer $(d r)$, and rer-rcr $(r r)$ contacts as functions of the homogeneity parameter $M^{*}$ and for $\alpha=0.5$. The error bars represent the standard deviation in the steady state.

located at the interface between the two phases. At the same time, $k_{d d}$ and $k_{r r}$ increase from 0.25 to 0.45 and from 0.2 to 0.55 , respectively. Interestingly, $k_{d d}>k_{r r}$ for $M^{*}<0.6$. In this way, as the segregation increases, the contact network passes from a contact network dominated by $d r$ contacts to a contact network dominated by $d d$ and $r r$ contacts. Note that we have $k_{d d} \simeq k_{r r}$ since we present results for $\alpha=0.5$. In general, $k_{d d}\left(M^{*}=0\right)$ declines from 0.8 for $\alpha=0.1$ to 0.05 for $\alpha=0.9$, whereas $k_{r r}\left(M^{*}=0\right)$ increases in the opposite proportion and $k_{d r}\left(M^{*}=0\right)$ remains between 0.2 and 0.5 , but the general trends observed in Fig. 13 are always the same.

Let us consider now the mean angular distributions, introduced in Sec. IV A, of the contact and force vectors supported by disk-disk, disk-rcrc, and rer-rcr contacts such that

$$
\begin{aligned}
P(\theta) & =P_{d d}(\theta)+P_{d r}(\theta)+P_{r r}(\theta), \\
\left\langle\ell_{n}\right\rangle(\theta) & =\left\langle\ell_{n}\right\rangle_{d d}(\theta)+\left\langle\ell_{n}\right\rangle_{d r}(\theta)+\left\langle\ell_{n}\right\rangle_{r r}(\theta), \\
\left\langle\ell_{t}\right\rangle(\theta) & =\left\langle\ell_{t}\right\rangle_{d d}(\theta)+\left\langle\ell_{t}\right\rangle_{d r}(\theta)+\left\langle\ell_{t}\right\rangle_{r r}(\theta), \\
\left\langle f_{n}\right\rangle(\theta) & =\left\langle f_{n}\right\rangle_{d d}(\theta)+\left\langle f_{n}\right\rangle_{d r}(\theta)+\left\langle f_{n}\right\rangle_{r r}(\theta), \\
\left\langle f_{t}\right\rangle(\theta) & =\left\langle f_{t}\right\rangle_{d d}(\theta)+\left\langle f_{t}\right\rangle_{d r}(\theta)+\left\langle f_{t}\right\rangle_{r r}(\theta),
\end{aligned}
$$

where the indices refer to the partial contributions of $d d$, $d r$, and $r r$ contacts. The corresponding anisotropies can be extracted by fitting each distribution; see Eq. (6). In practice, the anisotropy parameters are calculated through the force and fabrics tensors, presented in the Appendix by restricting the summation to each set of contacts. In principle, the principal directions of these partial angular distributions do not coincide with those of the overall tensors at all stages of shearing. But in the residual state the principal directions coincide so that the global anisotropy of each angular distribution is the sum of its partial anisotropies:

$$
a_{\gamma}=a_{\gamma}^{d d}+a_{\gamma}^{d r}+a_{\gamma}^{r r}
$$

where $\gamma$ stands alternatively for $\{c, \ell n, \ell t, f n, f t\}$. Note that by construction we have $a_{\ell t}^{d d}=0$.

Figure 14 shows the variation of the partial contact and force anisotropies averaged in the residual state for $\alpha=0.5$, as functions of the homogeneity parameter $M^{*}$, together with
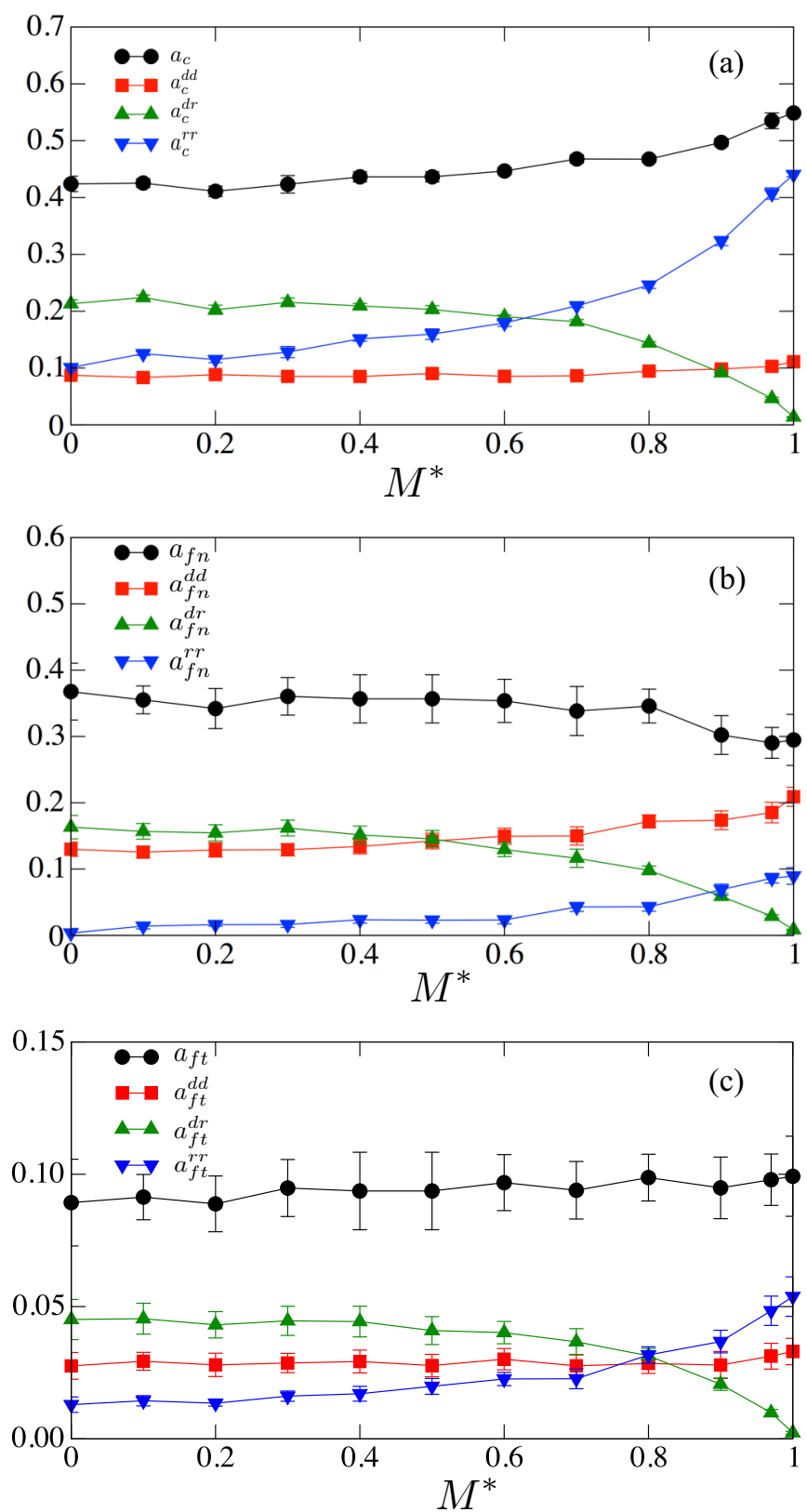

FIG. 14. Partial contact anisotropies $a_{c}^{d d}, a_{c}^{d r}$, and $a_{c}^{r r}$ (a), partial normal force anisotropies $a_{f n}^{d d}, a_{f n}^{d r}$, and $a_{f n}^{r r}(\mathrm{~b})$, and partial tangential force anisotropies $a_{f t}^{d d}, a_{f t}^{d r}$, and $a_{f t}^{r r}$ (c) of $d d, d r$, and $r r$ contacts as functions of the homogeneity parameter $M^{*}$, together with total anisotropies $a_{c}, a_{f n}$, and $a_{f t}$, for $\alpha=0.5$. The error bars represent the standard deviation in the steady state.

the total anisotropies $a_{c}, a_{f n}$, and $a_{f t}$. We do not consider here the normal and tangential branch anisotropies, which remain very small. We see that the contact and force anisotropies supported by $d d$ contacts remain constant as a function of $M^{*}$ although their proportion increases. This can be understood by remarking that the disk-disk contacts are not affected by the specificities of particle shapes (here elongation). In contrast, we see that all the partial contact, normal and tangential force anisotropies supported by $d r$ contacts decline with $M^{*}$, whereas the anisotropies supported by $r r$ contacts increase. 


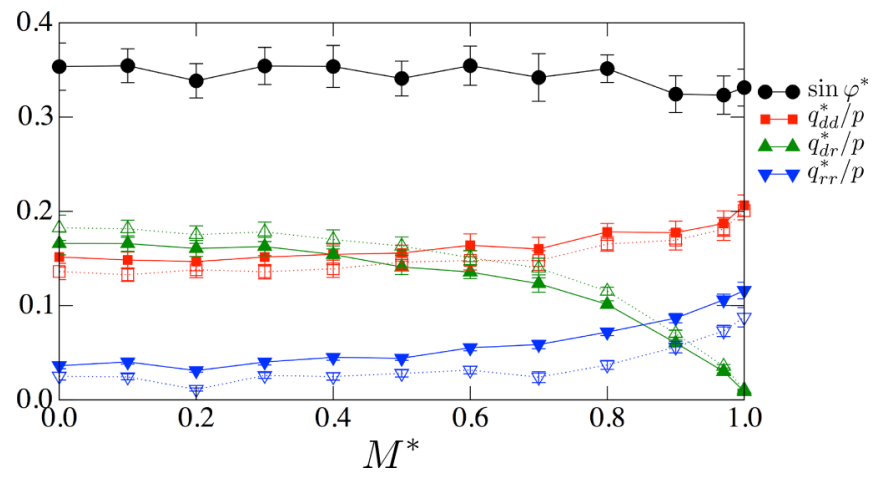

FIG. 15. Macroscopic friction angle $\sin \varphi^{*}$ and partial shear strengths for disk-disk $(d d)$, disk-rer $(d r)$, and rcrc-rcrc $(r r)$ contacts as functions of the homogeneity parameter $M^{*}$, together with the predicted values by Eq. (13) (empty symbols), for $\alpha=0.5$. The error bars represent the standard deviation in the steady state.

Here, it is remarkable that in all cases $a_{\gamma}^{d r}+a_{\gamma}^{r r}$ remains constant which, by virtue of Eq. (10) explains the fact that the global corresponding anisotropy remains also constant. In particular, Fig. 14(c) reveals also that for nearly segregated packings, friction is mainly mobilized at $r r$ contacts since $a_{f t}^{r r}>a_{f t}^{d r}+a_{f t}^{d d}$, which is in agreement with the observation done on Fig. 7(b).

\section{Stress partition}

Along the same line, the stress tensor can be partitioned as a sum of three tensors by grouping the contacts in Eq. (5) according to their types:

$$
\sigma=\sigma_{d d}+\sigma_{d r}+\sigma_{r r},
$$

where $\sigma_{d d}, \sigma_{d r}$, and $\sigma_{r r}$ are obtained from the expression of the stress tensor Eq. (5) by restricting the summation to $d d, d r$, and $r r$ contacts, respectively. The corresponding partial stress deviators $q_{d d}, q_{d r}$, and $q_{r r}$ are then calculated and normalized by the mean pressure $p$. The macroscopic friction angle is then given by

$$
\sin \varphi^{*}=\frac{q_{d d}^{*}}{p}+\frac{q_{d r}^{*}}{p}+\frac{q_{r r}^{*}}{p} .
$$

Figure 15 shows $q_{d d} / p, q_{d r} / p$, and $q_{r r} / p$ averaged in the residual state as a function of $M^{*}$ for $\alpha=0.5$. We see that $q_{d d} / p$ is nearly constant, and remarkably $q_{d r} / p$ follows a trend opposite to that of $q_{r r} / p$ so that the decrease of $q_{d r} / p$ with $M^{*}$ is exactly compensated by the increase of $q_{r r} / p$, which explains the independence of $\sin \varphi^{*}$ with respect to $M^{*}$. In particular, $q_{d r} / p \rightarrow 0$ for segregated packings, thus evidencing the critical role of contacts at the interface between the two species of particles. This confirms also the idea suggested in Sec. III that sliding is enhanced at the interface. From a broader point of view, fully segregated granular media are the archetypical representation of composite materials made of various layers and for which the interface between layers is known to behave often as a "weak zone" of the material $[72,73]$.

The harmonic partition of stresses presented in Sec. IV A holds also for the subnetwork of $d d, d r$, and $r r$ contacts, and one can show that

$$
\frac{q_{\xi}^{*}}{p} \simeq \frac{1}{2}\left(a_{c}^{\xi}+a_{\ell n}^{\xi}+a_{l t}^{\xi}+a_{f n}^{\xi}+a_{f t}^{\xi}\right),
$$

where $\xi$ stands alternatively for $\{d d, d r, r r\}$. This additive partition is nicely verified by our numerical data as shown in Fig. 15 in dashed lines. Thus the corresponding variations of the partial stresses with $M^{*}$ results from the corresponding variations of the associated partial anisotropies with $M^{*}$, underlying the role of each contact type in fabric, force transmission, and friction mobilization.

\section{CONCLUSIONS}

In this paper, a systematic numerical analysis of the quasistatic rheology of two-dimensional sheared granular mixtures was presented. We considered a binary mixture of disks and elongated particles of rounded-cap-rectangle shape (rcr). The binary mixture is characterized by the mixture ratio $\alpha$, which varies from 0 for a packing composed of only disks to 1 for a packing composed of only rcr particles, and with a homogeneity parameter $M^{*}$ which varies from 0 for an homogeneous packing to 1 for a fully segregated packing. The numerical samples were subjected to biaxial compression until a steady shear state was reached.

A major result of this work is that the shear strength is independent of the packing homogeneity and increases as the mixture ratio increases. In contrast, the packing fraction was found to be nearly independent from both $\alpha$ and $M^{*}$. We have also performed a detailed analysis of the microstructure. In particular, the fabric and force anisotropies were analyzed as a function of $\alpha$ and $M^{*}$.

The increase of the shear strength with respect to the mixture ratio appears to be a consequence of the increase of the contact network and normal force anisotropies whereas the increase of tangential anisotropy is compensated by a decrease of branch vector anisotropies. This behavior is explained by the fact that the elongated particles mobilize more friction than disks during flow and orient themselves orthogonally to the major principal stress direction. Replacing disks by rcr particles naturally enhances this phenomenon.

Remarkably, the contact and force anisotropies are independent with respect to the level of packing homogeneity, thus explaining also the independence of the shear strength. This finding is rather counterintuitive since, at least geometrically, the packing structure varies drastically with $M^{*}$. By performing an additive partition of the stress, force, and fabric anisotropies in terms of disk-disk, disk-rcr, and rcr-rcr contacts, we showed that the partial shear strength and anisotropies of rcr-rcr contacts increase with $M^{*}$, whereas those of disk-rcr contacts decrease. This is consistent with the observation that all local and global properties are independent of $M^{*}$.

This work is a systematic investigation of particle shape mixture ratio and the degree of mixture homogeneity by considering a binary mixture of different shapes. It shows that the mixture ratio parameter and, at first order, an "average homogeneity" parameter related to particle positions are good control parameters of the packings. Our findings highlight also the key role of the interface between two different species in a granular mixture, especially when the system is segregated. 
In this case, the strength supported by the contacts at the interfaces between the two species declines, revealing that the interface is a "weakness zone" of the material.

Hence an interesting issue suggested by this work is how the strength at the interface between two species can be increased. A natural way is by adding cohesive forces, which is typically the case for mixtures of wet and dry granular media, but we may expect that the weakness zones will then move from the interface to the zone where dry particles will be grouped. Thus, in view of realistic modeling of granular materials or, in a more general way, composite materials, we see here that the definition of a "good mixture" is not evident. The counterintuitive features evidenced in this work suggest the necessity of further investigations of the behavior of granular mixtures by considering other shapes, and also different interactions, both in $2 \mathrm{D}$ and $3 \mathrm{D}$. In particular, it would be highly instructive to assess the proper role of friction by varying this parameter systematically for each value of the homogeneity parameter.

\section{ACKNOWLEDGMENTS}

The authors acknowledge financial support by the Faculty of Engineering of Chiang Mai University. F.R. would also like to acknowledge the support of the ICoME2 Labex (No. ANR-11-LABX-0053) and the A*MIDEX projects (No. ANR-11-IDEX-0001-02) cofunded by the French program Investissements d'Avenir.

\section{APPENDIX: FORCE AND FABRIC TENSORS}

In practice, the values of all anisotropy parameters and the angles of corresponding privileged directions can be calculated from the following force and fabric tensors:

$$
\begin{aligned}
F_{\alpha \beta} & =\frac{1}{\pi} \int_{0}^{\pi} n_{\alpha} n_{\beta} P_{\theta}(\theta) d \theta, \\
\chi_{\alpha \beta}^{l n} & =\int_{0}^{\pi}\left\langle\ell_{n}\right\rangle(\theta) n_{\alpha} n_{\beta} P_{\theta}(\theta) d \theta, \\
\chi_{\alpha \beta}^{l t} & =\int_{0}^{\pi}\left\langle\ell_{t}\right\rangle(\theta) n_{\alpha} t_{\beta} P_{\theta}(\theta) d \theta, \\
\chi_{\alpha \beta}^{f n} & =\int_{0}^{\pi}\left\langle f_{n}\right\rangle(\theta) n_{\alpha} n_{\beta} P_{\theta}(\theta) d \theta, \\
\chi_{\alpha \beta}^{f t} & =\int_{0}^{\pi}\left\langle f_{t}\right\rangle(\theta) n_{\alpha} t_{\beta} P_{\theta}(\theta) d \theta,
\end{aligned}
$$

where $\alpha$ and $\beta$ design the components in the considered frame. From Eqs. (6) and (A1), assuming that in a biaxial sheared state $\theta_{c}=\theta_{f n}=\theta_{f t}=\theta_{\sigma}=\pi / 2, \theta_{l n}=\theta_{l t}=0$, the following relations are easily obtained:

$$
\begin{aligned}
a_{c} & =2\left(F_{1}-F_{2}\right) /\left(F_{1}+F_{2}\right), \\
a_{l n} & =2\left(\chi_{1}^{l n}-\chi_{2}^{l n}\right) /\left(\chi_{1}^{l n}+\chi_{2}^{l n}\right)-a_{c}, \\
a_{l t} & =2\left(\chi_{1}^{l}-\chi_{2}^{l}\right) /\left(\chi_{1}^{l}+\chi_{2}^{l}\right)-a_{c}-a_{l n}, \\
a_{f n} & =2\left(\chi_{1}^{f n}-\chi_{2}^{f n}\right) /\left(\chi_{1}^{f n}+\chi_{2}^{f n}\right)-a_{c}, \\
a_{f t} & =2\left(\chi_{1}^{f}-\chi_{2}^{f}\right) /\left(\chi_{1}^{f}+\chi_{2}^{f}\right)-a_{c}-a_{f n},
\end{aligned}
$$

where $\chi^{l}=\chi^{l n}+\chi^{l t}, \chi^{f}=\chi^{f n}+\chi^{f t}$, and the indices 1 and 2 refer to the principal values of each tensor. By construction, we have $\left(F_{1}+F_{2}\right)=1,\left(\chi_{1}^{l}+\chi_{2}^{l}\right)=\left\langle\ell_{n}\right\rangle$, and $\left(\chi_{1}^{f}+\chi_{2}^{f}\right)=$ $\left\langle f_{n}\right\rangle$. Note that $a_{c}, a_{f n}$, and $a_{f t}$ are always positive, whereas $a_{l n}$ and $a_{l t}$ are negative. Note that $a_{\ell t}$ is equal to 0 for packings composed of only disks.
[1] M. Pica Ciamarra, A. Coniglio, and M. Nicodemi, J. Phys.: Condens. Matter 17, S2549 (2005).

[2] S. Rémond, J. L. Gallias, and A. Mizrahi, Granular Matter 10, 157 (2008).

[3] B. Yohannes and K. M. Hill, Phys. Rev. E 82, 061301 (2010).

[4] Y. Fan, Y. Boukerkour, T. Blanc, P.-B. Umbanhowar, J.-M. Ottino, and R.-M. Lueptow, Phys. Rev. E 86, 051305 (2012).

[5] X. Yuan, N. Zheng, Q. Shi, G. Sun, and L. Li, Phys. Rev. E 87, 042203 (2013).

[6] Y. Fan and K. M. Hill, Phys. Rev. E 92, 022211 (2015).

[7] C. R. K. Windows-Yule, G. J. M. Douglas, and D. J. Parker, Phys. Rev. E 91, 032205 (2015).

[8] L. Staron and J. C. Phillips, Phys. Rev. E 92, 022210 (2015).

[9] K. M. Hill and J. Kakalios, Phys. Rev. E 52, 4393 (1995).

[10] T. Mullin, Science 295, 1851 (2002).

[11] P. M. Reis, T. Sykes, and T. Mullin, Phys. Rev. E 74, 051306 (2006).

[12] P.-G. Rognon, J.-N. Roux, M. Naaim, and F. Chevoir, Phys. Fluids 19, 058101 (2007).

[13] L. Staron and J. C. Phillips, Phys. Fluids 26, 033302 (2014).

[14] J. C. Williams, Powder Technol. 2, 13 (1968).

[15] J. Duran, J. Rajchenbach, and E. Clement, Phys. Rev. Lett. 70, 2431 (1993).
[16] J. B. Knight, H. M. Jaeger, and S. R. Nagel, Phys. Rev. Lett. 70, 3728 (1993).

[17] O. Pouliquen, J. Delour, and S. B. Savage, Nature (London) 386, 816 (1997).

[18] S. Aumaître, C. a. Kruelle, and I. Rehberg, Phys. Rev. E 64, 041305 (2001).

[19] J.-J. Moreau, Eur. J. Mech. A/Solids 13(4), 93 (1994).

[20] C.-R.-A. Abreu, F.-W. Tavares, and M. Castier, Powder Technol. 134, 167 (2003).

[21] S.-J. Roskilly, E.-A. Colbourn, O. Alli, D. Williams, K.-A. Paul, E.-H. Welfare, and P.-A. Trusty, Powder Technol. 203, 211 (2010).

[22] X.-D. Ma, Y.-B. Zhang, Y. Liu, and X.-W. Zheng, Granular Matter 18, 8 (2016).

[23] J.-C. Géminard and W. Losert, Phys. Rev. E 65, 041301 (2002).

[24] P. Jongchansitto, X. Balandraud, M. Grédiac, C. Beitone, and I. Preechawuttipong, Soft Matter 10, 8603 (2014).

[25] C. Voivret, F. Radjai, J.-Y. Delenne, and M. S. El Youssoufi, Phys. Rev. Lett. 102, 178001 (2009).

[26] D.-H. Nguyen, E. Azéma, P. Sornay, and F. Radjai, Phys. Rev. E 91, 032203 (2015).

[27] S. R. Williams and A. P. Philipse, Phys. Rev. E 67, 051301 (2003). 
[28] W. Man, A. Donev, F.-H. Stillinger, M. T. Sullivan, W. B. Russel, D. Heeger, S. Inati, S. Torquato, and P. M. Chaikin, Phys. Rev. Lett. 94, 198001 (2005).

[29] A. Donev, R. Connelly, F.-H. Stillinger, and S. Torquato, Phys. Rev. E 75, 051304 (2007).

[30] S. Torquato and Y. Jiao, Phys. Rev. E 80, 041104 (2009).

[31] A. V. Kyrylyuk, A. Wouterse, and A. P. Philipse, in Random Packings of Rod-sphere Mixtures Simulated by Mechanical Contraction, AIP Conf. Proc. No. 1145 (AIP, New York, 2009), p. 211.

[32] E. Azéma and F. Radjaï, Phys. Rev. E 81, 051304 (2010).

[33] Y. Jiao, F. H. Stillinger, and S. Torquato, Phys. Rev. E 81, 041304 (2010).

[34] Y. Jiao and S. Torquato, Phys. Rev. E 84, 041309 (2011).

[35] E. Azéma and F. Radjaï, Phys. Rev. E 85, 031303 (2012).

[36] E. Azéma, N. Estrada, and F. Radjaï, Phys. Rev. E 86, 041301 (2012).

[37] M. Boton, E. Azéma, N. Estrada, F. Radjaï, and A. Lizcano, Phys. Rev. E 87, 032206 (2013).

[38] E. Azéma, F. Radjaï, B. Saint-Cyr, J.-Y. Delenne, and P. Sornay, Phys. Rev. E 87, 052205 (2013).

[39] E. Azéma, F. Radjai, and F. Dubois, Phys. Rev. E 87, 062203 (2013).

[40] A.-G. Athanassiadis, M.-Z. Miskin, P. Kaplan, N. Rodenberg, S.-H. Lee, J. Merritt, E. Brown, J. Amend, H. Lipson, and H. Jaeger, Soft Matter 10, 48 (2014).

[41] CEGEO, B. Saint-Cyr, K. Szarf, C. Voivret, E. Azéma, V. Richefeu, J.-Y. Delenne, G. Combe, C. Nouguier-Lehon, P. Villard et al., Eur. Phys. Lett. 98, 44008 (2012).

[42] I. Zuriguel, T. Mullin, and J. M. Rotter, Phys. Rev. Lett. 98, 028001 (2007).

[43] R.-C. Hidalgo, I. Zuriguel, D. Maza, and I. Pagonabarraga, Phys. Rev. Lett. 103, 118001 (2009).

[44] G. W. Delaney, J. E. Hilton, and P. W. Cleary, Phys. Rev. E 83, 051305 (2011).

[45] S. Wegner, T. Börzsönyi, T. Bien, G. Rose, and R. Stannarius, Soft Matter 8, 10950 (2012).

[46] T. Börzsönyi, B. Szabó, S. Wegner, K. Harth, J. Török, E. Somfai, T. Bien, and R. Stannarius, Phys. Rev. E 86, 051304 (2012).

[47] T. Börzsönyi and R. Stannarius, Soft Matter 9, 7401 (2013).

[48] F. S. Lai, Powder Technol. 24, 73 (1979).

[49] A. A. Alhwaige, S. M. Tasirin, A. M. Sowedan, W. Ramli, and W. Daud, in Proceedings of the World Congress on Engineering and Computer Science 2008, WCECS 2008, San Francisco, USA, 2008, http://www.iaeng.org/ publication/WCECS2008/WCECS2008_pp118-122.pdf.

[50] M. Jean, Comput. Methods Appl. Mech. Eng. 177, 235 (1999).

[51] F. Radjai and V. Richefeu, Mech. Mater. 41, 715 (2009).

[52] F. Radjaï and F. Dubois, Modélisation Numérique Discrète Des Matériaux Granulaires (Hermès, Lavoisier, 2010).

[53] A. Donev, F.-H. Stillinger, P. M. Chaikin, and S. Torquato, Phys. Rev. Lett. 92, 255506 (2004).

[54] G. Delaney, D. Weaire, S. Hutzler, and S. Murphy, Philos. Mag. Lett. 85, 89 (2005).

[55] C. Nouguier-Lehon, C. R. Mécanique 338, 587 (2010).

[56] C. Voivret, F. Radjaï, J.-Y. Delenne, and M. S. El Youssoufi, Phys. Rev. E 76, 021301 (2007).

[57] G. D. R. Midi, Eur. Phys. J. E 14, 341 (2004).

[58] L. Staron, F. Radjai, and J.-P. Vilotte, Eur. Phys. J. E 18, 311 (2005).

[59] J. Mitchell and K. Soga, Fundamentals of Soil Behavior (Wiley, New York, 2005).

[60] J. Lee, M. Guimaraes, and J.-C. Santamarina, J. Geotech. Geoenviron. Eng. 133, 1136 (2007).

[61] J.-C. Santamarina and H. Shin, in Meso-Scale Shear Physics in Earthquake and Landslide Mechanics (CRC Press, Boca Raton, FL, 2009), pp. 159-190.

[62] F. Radjai, D.-E. Wolf, M. Jean, and J.-J. Moreau, Phys. Rev. Lett. 80, 61 (1998).

[63] H. Troadec, F. Radjai, S. Roux, and J. C. Charmet, Phys. Rev. E 66, 041305 (2002).

[64] N. P. Kruyt, Int. J. Solids Struct. 40, 3537 (2003).

[65] S. J. Antony and N. P. Kruyt, Phys. Rev. E 79, 031308 (2009).

[66] N.-P. Kruyt, Mech. Mater. 44, 120 (2012).

[67] L. Rothenburg and R.-J. Bathurst, Geotechniques 39, 601 (1989).

[68] N.-P. Kruyt and L. Rothenburg, Acta Mech. 225, 2301 (2014).

[69] H. Ouadfel and L. Rothenburg, Mech. Mater. 33, 201 (2001).

[70] E. Azéma, F. Radjai, and G. Saussine, Mech. Mater. 41, 729 (2009).

[71] M. Acevedo, R.-C. Hidalgo, I. Zuriguel, D. Maza, and I. Pagonabarraga, Phys. Rev. E 87, 012202 (2013).

[72] R.-M. Jones and K.-S. Devens, Mechanics of Composite Materials With Large-Scale Curving of Filler (Taylor and Francis, Inc., London, 1983), Vol. 18.

[73] Advances in Materials Science and Engineering, edited by A. Kelly (Elsevier, Amsterdam, 1994). 